Bo-Yang Ma, Tao Fu, Yi Jin, Ping Wei, Ji-Xing Zhao and Li Zhao*

\title{
Synthesis and crystal structure of 1-\{4-[(2-bromo- 6-hydroxy-benzylidene)amino]phenyl\}ethanone, $\mathrm{C}_{15} \mathrm{H}_{12} \mathrm{BrNO}_{2}$
}

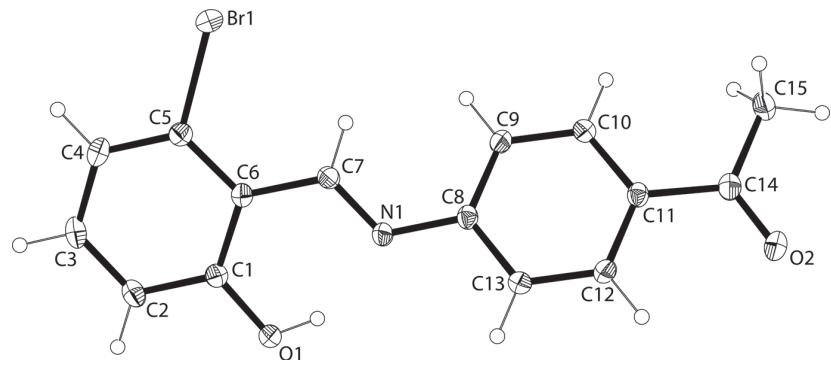

https://doi.org/10.1515/ncrs-2020-0182

Received April 11, 2020; accepted April 30, 2020; available

online June 18, 2020
Table 1: Data collection and handling.

\begin{tabular}{ll}
\hline Crystal: & Orange block \\
Size: & $0.17 \times 0.15 \times 0.14 \mathrm{~mm}$ \\
Wavelength: & Mo $K \alpha$ radiation $(0.71073 \AA$ $)$ \\
$\mu:$ & $3.11 \mathrm{~mm}^{-1}$ \\
Diffractometer, scan mode: & Bruker D8 Venture, $\varphi$ and $\omega$ \\
$\theta_{\text {max }}$, completeness: & $26.0^{\circ},>99 \%$ \\
$N\left(h k l_{\text {measured }}, N(h k l)_{\text {unique }}, R_{\text {int }}:\right.$ & $24608,2593,0.049$ \\
Criterion for $I_{\text {obs }}, N(h k l)_{\text {gt }}:$ & $I_{\text {obs }}>2 \sigma\left(I_{\text {obs }}\right), 2288$ \\
$N(\text { param })_{\text {refined }}:$ & 174 \\
Programs: & Bruker [1], SHELX [2], Olex2 [3]
\end{tabular}

Table 2: Fractional atomic coordinates and isotropic or equivalent isotropic displacement parameters $\left(\AA^{2}\right)$.

\begin{tabular}{lrrrr}
\hline Atom & $\boldsymbol{x}$ & $\boldsymbol{y}$ & $\boldsymbol{z}$ & $\boldsymbol{U}_{\text {iso }} / \boldsymbol{U}_{\text {eq }}$ \\
\hline Br1 & $0.82593(2)$ & $0.95481(2)$ & $0.08527(2)$ & $0.03478(8)$ \\
O1 & $0.62308(17)$ & $0.56026(9)$ & $0.06731(10)$ & $0.0314(3)$ \\
H1 & 0.577341 & 0.584163 & 0.011712 & $0.047^{*}$ \\
O2 & $0.15838(18)$ & $0.68675(11)$ & $-0.52281(10)$ & $0.0382(3)$ \\
N1 & $0.55229(18)$ & $0.69284(11)$ & $-0.07795(10)$ & $0.0246(3)$ \\
C1 & $0.7123(2)$ & $0.63642(13)$ & $0.11662(13)$ & $0.0246(4)$ \\
C2 & $0.8001(2)$ & $0.61139(15)$ & $0.21062(14)$ & $0.0309(4)$ \\
H2 & 0.796031 & 0.542084 & 0.237720 & $0.037^{*}$ \\
C3 & $0.8927(2)$ & $0.68739(16)$ & $0.26394(14)$ & $0.0326(4)$ \\
H3 & 0.952612 & 0.669828 & 0.327582 & $0.039^{*}$ \\
C4 & $0.8995(2)$ & $0.78922(15)$ & $0.22595(14)$ & $0.0309(4)$ \\
H4 & 0.962500 & 0.841342 & 0.263605 & $0.037^{*}$ \\
C5 & $0.8138(2)$ & $0.81384(14)$ & $0.13310(13)$ & $0.0251(4)$ \\
C6 & $0.7197(2)$ & $0.73897(13)$ & $0.07469(12)$ & $0.0225(3)$ \\
C7 & $0.6365(2)$ & $0.76292(13)$ & $-0.02606(13)$ & $0.0238(4)$ \\
H7 & 0.644513 & 0.831659 & -0.054027 & $0.029^{*}$ \\
C8 & $0.4680(2)$ & $0.71653(13)$ & $-0.17516(12)$ & $0.0234(4)$ \\
C9 & $0.4060(2)$ & $0.81582(14)$ & $-0.20102(13)$ & $0.0266(4)$ \\
H9 & 0.424527 & 0.872686 & -0.153837 & $0.032^{*}$ \\
C10 & $0.3175(2)$ & $0.83162(14)$ & $-0.29519(13)$ & $0.0267(4)$ \\
H10 & 0.275808 & 0.899605 & -0.312454 & $0.032^{*}$ \\
C11 & $0.2886(2)$ & $0.74903(14)$ & $-0.36536(12)$ & $0.0238(4)$ \\
C12 & $0.3525(2)$ & $0.65021(14)$ & $-0.33948(13)$ & $0.0274(4)$ \\
H12 & 0.335122 & 0.593601 & -0.387096 & $0.033^{*}$ \\
C13 & $0.4409(2)$ & $0.63362(14)$ & $-0.24527(13)$ & $0.0269(4)$ \\
H13 & 0.483310 & 0.565752 & -0.228215 & $0.032^{*}$ \\
& & & &
\end{tabular}

*Corresponding author: Li Zhao, School of Chemical and Biological Engineering, Lanzhou Jiaotong University, Lanzhou 730070, P.R. China, e-mail: zhaoli_72@163.com. https://orcid.org/00000002-9898-9552

Bo-Yang Ma, Tao Fu, Yi Jin and Ping Wei: Lanzhou Jiaotong University, School of Chemical and Biological Engineering, Lanzhou 730070, P.R. China

Ji-Xing Zhao: Shihezi University, Analysis and Testing Center, Xinjiang 832003, P.R. China 
Table 2 (continued)

\begin{tabular}{lrrrr}
\hline Atom & $\boldsymbol{x}$ & $\boldsymbol{y}$ & $\boldsymbol{z}$ & $\boldsymbol{U}_{\text {iso }}{ }^{*} / \boldsymbol{U}_{\text {eq }}$ \\
\hline C14 & $0.1855(2)$ & $0.76170(15)$ & $-0.46556(13)$ & $0.0273(4)$ \\
C15 & $0.1170(3)$ & $0.86800(16)$ & $-0.49427(15)$ & $0.0357(4)$ \\
H15A & 0.207631 & 0.915070 & -0.510291 & $0.054^{*}$ \\
H15B & 0.059597 & 0.896639 & -0.435766 & $0.054^{*}$ \\
H15C & 0.038865 & 0.861769 & -0.555529 & $0.054^{*}$ \\
\hline
\end{tabular}

1-(4-amino-phenyl)ethanone (135.16 mg, $1 \mathrm{mmol}$ ) at $328 \mathrm{~K}$. After $10 \mathrm{~h}$, the mixture was filtered. The filtrate was recrystallized, and several clear light orange crystals were obtained. Anal. Calcd. for $\mathrm{C}_{15} \mathrm{H}_{12} \mathrm{BrNO}_{2}$ : C, 56.62\%; H, 3.80\%; N, 4.40\%. Found: C, 56.93\%; H, 3.89\%; N, 4.25\%.

\section{Experimental details}

Hydrogen atoms were placed in their geometrically idealized positions and constrained to ride on their parent atoms.

\section{Comment}

Schiff bases attract great attention due to their easy preparation, diversity and structural variability $[7,8]$. They play a significant role in development of the chelate ligands in the field of coordination chemistry $[9,10]$. Schiff bases and their metal complexes are widely studied due to various physical properties [11-13], supramolecular aspects [14-16], luminescence [17] and so on.

The single crystal structure of the title compound was determined by X-ray crystallography. In the title compound structure, there is a strong intramolecular $\mathrm{O} 1-\mathrm{H} 1 \cdots \mathrm{N} 1$ hydrogen bond interaction $(\mathrm{d}(\mathrm{N} 1 \cdots \mathrm{H} 1)=1.804 \AA$, $\mathrm{d}(\mathrm{O} 1-$

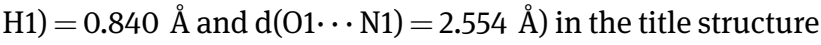
(cf. the figure). All geometric parameters are in the typical ranges [4-8].

\section{References}

1. Bruker. APEX2, SAINT and SADABS. Bruker AXS Inc., Madison, WI, USA (2012)

2. Sheldrick, G. M.: A short history of SHELX. Acta Crystallogr. A64 (2008) 112-122.

3. Dolomanov, O. V.; Bourhis, L. J.; Gildea, R. J.; Howard, J. A. K.; Puschmann, H.: OLEX2: a complete structure solution, refinement and analysis program. J. Appl. Crystallogr. 42 (2009) 339-341.

4. Zhao, J. X.; Zhao, L.; Li, P. P.; Wang, F.; An, Q. Q.: Crystal structure of $(E)-1-(4-((E)$-5-bromo-2-hydroxybenzylidene) amino)phenyl)ethan-1-one $O$-methyl oxime, $\mathrm{C}_{16} \mathrm{H}_{15} \mathrm{BrN}_{2} \mathrm{O}_{2}$. Z. Kristallogr. NCS 232 (2017) 731-732.

5. Ma, J. X.; Wei, P.; Li, Q. L.; Fu, T.; Zhao, J. X.; Zhao, L.: Crystal structure of (E)-1-(4-(((E)-2-bromo-6-hydroxybenzylidene) amino)phenyl)ethan-1-one $O$-methyl oxime, $\mathrm{C}_{16} \mathrm{H}_{15} \mathrm{BrN}_{2} \mathrm{O}_{2}$. Z. Kristallogr. NCS 235 (2020) 19-20.

6. Li, P.-P.; Ma, J.-X.; Li, Q.-L.; Zhao, J.-X.; Zhao, L.: Crystal structure of 1-\{4-[(2-hydroxy-5-methyl benzylidene)amino]phenyl\} ethanone $O$-ethyl-oxime, $\mathrm{C}_{18} \mathrm{H}_{20} \mathrm{~N}_{2} \mathrm{O}_{2}$. Z. Kristallogr. NCS 233 (2018) 759-761.

7. Zhao, L.; Zhao, J. X.; An, Q. Q.; Wang, F.: Crystal structure of (E)1-(4-((E)-3,5-dibromo-2-hydroxybenzylidene)amino)phenyl) ethan-1-one $O$-methyl oxime, $\mathrm{C}_{17} \mathrm{H}_{16} \mathrm{Br}_{2} \mathrm{~N}_{2} \mathrm{O}_{2}$. Z. Kristallogr. NCS 231 (2016) 1053-1054.

8. Fu, T.; Ma, J. X.; Li, Q. L.; Wei, P.; Zhao, J. X.; Zhao, L.: Crystal structure of (E)-1-\{4-[(4-fluoro-2-hydroxybenzylidene)amino] phenyl\}ethanone $O$-methyl oxime, $\mathrm{C}_{16} \mathrm{H}_{15} \mathrm{FN}_{2} \mathrm{O}_{2}$. Z. Kristallogr. NCS 235 (2020) 293-295.

9. Sun, Y. X.; Lu, R. E.; Li, X. R.; Zhao, Y. Y.; Li, C. Y.: A Schiff base ligand containing oxime group and its $\mathrm{Cu}$ (II) complex: syntheses and supramolecular structures. Chin. J. Inorg. Chem. 31 (2015) 1055-1062.

10. Sun, Y. X.; Li, C. Y.; Yang, C. J.; Zhao, Y. Y.; Guo, J. Q.; Yu, B.: Two $\mathrm{Cu}(\mathrm{II})$ complexes with Schiff base ligands: synthesis, crystal structure, spectroscopic properties, and substituent effect. Chin. J. Inorg. Chem. 32 (2016) 327-335.

11. Sun, Y. X.; Zhao, Y. Y.; Li, C. Y.; Yu, B.; Guo, J. Q.; Li, J.: Supramolecular cobalt(II) and copper(II) complexes with Schiff base ligand: syntheses, characterizations and crystal structures. Chin. J. Inorg. Chem. 32 (2016) 913-920.

12. Zhao, L.; Dong, X. T.; Sun, Y. X.; Cheng, Q.; Dong, X. Y.; Wang, L.: Synthesis, crystal structure and thermal property of a 1D chain-like copper(II) complex. Chin. J. Inorg. Chem. 28 (2012) 2413-2418.

13. Li, P. P.; Zhao, L.; Zhao, J. X.; Zhu, Z. B.; Wang, F.; An, Q. Q.: Synthesis and crystal structure of bis\{1-(((4-(1-(hydroxyimino)ethyl) phenyl)imino)methyl) naphthalen-2-olato- $\left.\mathrm{K}^{2} \mathrm{~N}, 0\right\}$ copper(II), $\mathrm{C}_{38} \mathrm{H}_{30} \mathrm{CuN}_{4} \mathrm{O}_{4}$. Z. Kristallogr. NCS 232 (2017) 889-890.

14. Yu, B.; Sun, Y. X.; Yang, C. J.; Guo, J. Q.; Li, J.: Synthesis and crystal structures of an unexpected tetranuclear zinc(II) complex and a benzoquinone compound derived from $\mathrm{Zn}$ - and Cd" - promoted reactivity of Schiff base ligands. Z. Anorg. Allg. Chem. 643 (2017) 689-698.

15. Liu, P. P.; Wang, C. Y.; Zhang, M.; Song, X. Q.: Pentanuclear

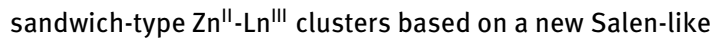
salicylamide ligand: structure, near-infrared emission and magnetic properties. Polyhedron 129 (2017) 133-140.

16. Chang, J.; Zhang, H. J.; Jia, H. R.; Sun, Y. X.: Binuclear nickel(II) and zinc(II) complexes based on 2-amino-3-hydroxy-pyridine Schiff base: syntheses, supramolecular structures and spectral properties. Chin. J. Inorg. Chem. 34 (2018) 2097-2107.

17. Dong, W. K.; Ma, J. C.; Zhu, L. C.; Sun, Y. X.; Akogun, S. F.; Zhang, Y.: A series of heteromultinuclear zinc(II)-lanthanide(III) complexes based on 3-MeOsalamo: syntheses, structural characterizations, and luminescent properties. Cryst. Growth Des. 16 (2016) 6903-6914. 